\title{
CHARACTERISTICS OF PATIENTS WHO UNDERWENT LOWER LIMB AMPUTATION DUE TO CRITICAL LIMB ISCHEMIA - ONE CENTER EXPERIENCE
}

\author{
CHARAKTERYSTYKA PACJENTÓW PODDANYCH AMPUTACJI \\ Z POWODU PRZEWLEKŁEGO NIEDOKRWIENIA KOŃCZYN DOLNYCH \\ - DOŚWIADCZENIE JEDNEGO OŚRODKA
}

\begin{abstract}
${ }^{1}$ Student Scientific Group, Chair and Clinic of Vascular and Internal Diseases, Faculty of Health Sciences, Ludwik Rydygier Collegium Medicum in Bydgoszcz, Nicolaus Copernicus University in Toruń ${ }^{2}$ Clinic of Vascular and Internal Diseases, Jan Biziel University Hospital No. 2, Bydgoszcz ${ }^{3}$ Chair of Vascular and Internal Diseases, Faculty of Health Sciences, Ludwik Rydygier Collegium Medicum in Bydgoszcz, Nicolaus Copernicus University in Toruń, Ludwik Rydygier Collegium Medicum in Bydgoszcz
\end{abstract}

\section{S u m m a r y}

Introduction. The aim of this study is to analyze the characteristics of patients in whom leg amputation was performed due to critical limb ischemia (CLI).

Material and methods. Retrospective analysis of the characteristics of patients who underwent leg amputation between 2012 and 2014 in our center.

R e s u $1 \mathrm{ts}$. Between 2012 and 2014, 39 amputations of 37 limbs were performed on 32 patients treated due to CLI. Amputations amounted to $39 / 1282(3.2 \%)$ of all vascular procedures performed on the lower limbs. They concerned, respectively: forefoot $-49 \%$, calf $-15 \%$, and thigh $-36 \%$. In $85 \%$ of patients, the amputations were preceded by vascular procedures. The median length of time between the first revascularization procedure and amputation amounted to 99 \pm 8 -1088 days. Patients who underwent primary amputation $(\mathrm{n}=6,15 \%)$ compared to the individuals with secondary amputation were treated less frequently with aspirin (67\% vs. $97 \% ; \mathrm{p}=0.01)$ and statins ( $50 \%$ vs. $94 \%, \mathrm{p}=0.002)$. Patients who underwent amputation at thigh level compared with subjects with forefoot amputation were hospitalized for a significantly longer period of time, diagnosed more often with chronic heart failure (CHF) and chronic kidney disease (CKD), and were more often tobacco smokers.

Conclusions. The main factors determining the need for amputation, its level and time between the first revascularization procedure and leg amputation were: the number of preceding procedures and stent implantations, comorbidity of $\mathrm{CHF}$ and $\mathrm{CKD}$, smoking habit, inadequate pharmacotherapy (probably due to delayed diagnosis), severity of angiographic lesions, and higher MPV and INR values.

\section{Streszczenie}

W s t ę p. Celem niniejszej pracy było przedstawienie charakterystyki pacjentów poddanych amputacji kończyn dolnych $\mathrm{z}$ powodu krytycznego niedokrwienia (CLI) $\mathrm{w}$ jednym ośrodku angiologicznym.

Pacjenci i metody. W latach 2012-2014 w klinice wykonano 39 amputacji kończyn dolnych, na 37 kończynach, u 32 pacjentów leczonych z powodu CLI.

Wyniki. Amputacje kończyn dolnych stanowiły $39 / 1282$ (3\%) wszystkich procedur naczyniowych na tętnicach kończyn dolnych. 49\% amputacji dotyczyło przodostopia, $15 \%$ podudzia oraz $36 \%$ uda; $85 \%$ amputacji przeprowadzono na kończynach, które wcześniej były rewaskularyzowane. Średni czas między pierwszym zabiegiem naczyniowym a amputacją kończyny wynosił $99 \pm$ 8-1088 dni. Pacjenci poddani pierwotnej amputacji rzadziej przyjmowali aspirynę $(67 \%$ vs. $94 \% ; \mathrm{p}=0,01)$ oraz statyny (50\% vs. 94\%, p=0,002). Pacjenci $z$ amputacją na udzie, $\mathrm{w}$ porównaniu $\mathrm{z}$ osobami $\mathrm{z}$ amputowanym przodostopiem, 
byli w związku z zabiegiem dłużej hospitalizowani, częściej mieli rozpoznaną przewlekłą niewydolność serca (PNS) i nerek (PNN), częściej palili i rzadziej przebyli udar mózgu. Nie stwierdzono różnic częstości występowania cukrzycy i dyslipidemii oraz nasilenia zmian angiograficznych. Zakres amputacji korelował m.in $\mathrm{z}$ wcześniejszą diagnozą PNS i PNN, przebyciem udaru mózgu, wartością MPV, INR i kreatyniny. Czas między wykonanym zabiegiem naczyniowym a amputacją korelował znamiennie m.in. z: liczbą wykonanych zabiegów i stentów implantowanych podczas pierwszego zabiegu.
W n i o ski. 15\% pacjentów z CLI trafiało do szpitala w stanie uniemożliwiającym podjęcie zabiegu rewaskularyzacyjnego. Czynnikami determinującymi konieczność amputacji i jej zakres były: liczba wcześniejszych zabiegów i implantacji stentów, współistniejące PNS i PNN, palenie tytoniu, nieadekwatna farmakoterapia (opóźnienie diagnozy?), nasilenia zmian angiograficznych oraz wyższe wartości MPV i INR; nie wpływały na nią natomiast cukrzyca i dyslipidemia.

Key words: lower limb ischemia, critical limb ischemia, lower limb amputation, risk factors

Stowa kluczowe: przewlekłe niedokrwienie kończyn dolnych, krytyczne niedkrwienie kończyn dolnych, amputacja kończyn dolnych, czynniki ryzyka

\section{INTRODUCTION}

There is a great deal of evidence indicating that primary amputation is still one of the main procedures in acute as well as chronic limb ischemia treatment. In some countries, an amputation is the first choice of treatment in as many as $67 \%$ of patients with critical limb ischemia (CLI). In this group, $31.8-50 \%$ of patients undergo leg amputation without an earlier diagnosis [1]; arteriography is performed in $16 \%$ of patients, the ankle-brachial index (ABI) is measured in $35 \%$ of patients, and only $26 \%$ consult a cardiologist and $21 \%$ - a vascular surgeon. Perioperative mortality rate related to limb amputation in the US ranges from 4 to $30 \%$ in different institutions and the 12-month rate increases to $45 \%$. These data show that peripheral artery disease (PAD) is still not diagnosed and managed correctly.

According to Yost, approximately 65-75 thousand major amputations (above and below the knee) are performed annually due to CLI [2]. The annual direct cost of CLI treatment in the US exceeds $\$ 25$ billion. Furthermore, the 5-year mortality rate of $70 \%$ for patients with CLI means that the number of deaths attributed to CLI is higher than the total of deaths due to coronary artery disease (CAD), breast cancer and colorectal cancer over the same period. To the previously mentioned health and social consequences of lower limb amputation, the costs of prosthetic, orthopedic, rehabilitation and work absence should also be added. However, it seems that proper prevention and management of PAD might reduce the impact of these health and social consequences, limit the use of public health system resources, and improve the quality of life not only for the patients, but also for their families. All the above-mentioned data show that the need for leg amputation in patients with PAD is a critical issue. Therefore, any investigation which might help to identify the risk factors for leg amputation, both primary and secondary, is justified and necessary. These types of investigation could help to introduce a strategy for leg amputation prevention based on risk factor stratification. As these risk factors characterize patients with primary and secondary leg amputation, we conducted our study to identify them in our own material.

\section{MATERIAL AND METHODS}

Clinical data related to 41 lower limb amputations performed between 2012 and 2014 in our clinic were retrospectively analyzed.

We were given a permission to conduct the analysis by the local Bioethical Commission on 02.18.2014 in accordance with an annex from 04.21.2015. The study was conducted in accordance with the Revised Declaration of Helsinki.

Statistical analysis was performed with STATISTICA software for Windows 10.0 (StatSoft, Inc. 2011, STATISTICA, data analysis software system, version 10). The results are expressed as mean \pm standard deviation. Non-parametric tests were used to compare the values between the groups due to the inability to refute the hypothesis of the absence of a normal distribution (Shapiro-Wilk test): the MannWhitney $U$ test and the Fisher's exact test. The Spearman's rank correlations were checked. The variables determining the time duration between the first revascularization procedure and the amputation were analyzed using progressive stepwise regression. The statistical significance level was set at a P-value $<0.05$. 


\section{RESULTS}

During 2012 and 2014, 41 lower limb amputations were performed in our clinic. They involved 39 limbs of 32 patients with CLI. During this time, 8285 patients were treated in our clinic, of whom 1437 (17.3\%) were treated with any intervention. Of these interventions, $1010 / 1437(70.3 \%)$ were endovascular, of which $673 / 1010(66.7 \%)$ concerned lower extremities. The remaining operations included 240/1437 (16.7\%) surgical procedures, among which 85/240 (35.4\%) concerned lower limb vascular procedures, and 187/1437 (13\%) hybrid procedures, which combined endovascular and surgical methods on leg vessels. Lower limb amputations amounted to $41 / 240$ (17\%) of all surgical procedures and 41/945 (4.3\%) of all procedures concerning the vessels of the lower limbs. Among the amputations analyzed, 21/41 (51\%) concerned the forefoot, $6 / 41(15 \%)$ the calf and $14 / 41$ (34\%) the thigh. The reasons for amputation were as follows: in 18 cases (44\%), extensive necrosis or its progression, in 21 cases $(51 \%)$ infected necrosis, and in two $(5 \%)$ cases the lack of wound healing after less extensive amputation. None of the patients experienced a cardiovascular event during hospitalization or in the 30 days after being discharged home.

One patient had three successive amputations of the same leg during the same hospitalization. In the subsequent analysis, this patient was counted as an individual with thigh level amputation. This gives 39 amputations overall, as shown in Table 1. Thus, 39 leg amputation procedures during separate hospitalizations are included in the analysis presented in Table 1. In six (15\%) patients, leg amputation was conducted as a primary procedure (on a limb that had not previously been revascularized), and in the remaining 33 (85\%) amputations were performed on a previously revascularized limb due to CLI (Rutherford class 4-6), where revascularizations were both endovascular $(100 \%)$ and surgical $(13 / 33 ; 39 \%)$. Each of the 39 amputation procedures analyzed had been treated with vascular procedures between one and five times, which resulted in a total of 54 interventions. The average time between the first procedure on the limb and amputation amounted to $220.4 \pm 272.7$ days (median 99 days, range: 8-1088 days).

Patients who underwent primary amputation were not significantly different from the patients who had been treated with vascular procedures before the amputation (secondary amputation) when it came to the following: length of hospitalization due to amputation, the level of the amputation, a prior amputation on other lower limb, the existence of infected necrosis or diabetes mellitus prevalence (Table 1). There had also not been any significant differences in the concentration of glucose, low-density lipoprotein (LDL) cholesterol, high-density lipoprotein (HDL) cholesterol, triglycerides or creatinine in the blood (Table 1). However, in the group of patients with a primary amputation, left lower limb amputation was significantly more frequent and the patients were slightly younger. Moreover, they took aspirin, clopidogrel and statins less often than individuals with a secondary leg amputation.

Next, we analyzed factors affecting the level of the leg amputation. The patients who had a thigh-level amputation $(\mathrm{n}=14 / 39,36 \%)$ were hospitalized significantly longer $(15.1 \pm 6.5$ days vs. $7.2 \pm 5.2$ days $)$ compared to the patients who underwent a forefootlevel amputation $(\mathrm{n}=20 / 39,51 \%)$ (Table 2). The group of patients with a thigh-level amputation was significantly older, more often diagnosed with chronic heart failure (CHF) and chronic kidney disease (CKD). Smoking was not significantly less prevalent among thigh-level amputees (43\% vs 67\%) and this group was more likely to have a history of stroke (36\% vs 5\%) (Table 2). They also had higher mean platelet volume (MPV), greater platelet distribution width (PDW) and a higher creatinine level. However, diabetes mellitus, blood glucose, LDL and HDL cholesterol, and triglycerides were similar in both groups. There was also no difference in initial angiographic lesion severity at the aortoiliac femoropopliteal level below the knee, or on the foot. Patients with a thigh-level amputation had had significantly more stents implanted before the amputation, the full length of these stents being significantly longer than in other patients.

Following univariate analysis (Spearman's rank correlation), the level of leg amputation (forefoot, calf, or thigh) correlated significantly with: length of hospitalization after amputation $(\mathrm{R}=0.56 ; \mathrm{p}=0.0002)$; previous diagnosis of $\mathrm{CHF}(\mathrm{R}=0.34 ; \mathrm{p}=0.032)$; history of stroke $(\mathrm{R}=0.36 ; \mathrm{p}=0.023)$; MPV value $(\mathrm{R}$ $=0.45 ; \mathrm{p}=0.0044)$; international normalized value (INR) value $(\mathrm{R}=0.38 ; \mathrm{p}=0.019)$; history of chronic obstructive lung disease $(\mathrm{COLD})(\mathrm{R}=0.46 ; \mathrm{p}=$ $0.0033)$; and creatinine level $(\mathrm{R}=0.33 ; \mathrm{p}=0.040)$.

However, the time between the first vascular procedure and amputation of this leg was closely related to the number of vascular procedures before the amputation $(\mathrm{R}=0.64 ; \mathrm{p}=0.0001)$, hemoglobin value $(\mathrm{R}=-0.37 ; \mathrm{p}=0.035)$, leucocyte blood count $(\mathrm{R}=-$ $0.35 ; \mathrm{p}=0.047)$, and the number of stents implanted during the first procedure $(\mathrm{R}=0.36 ; \mathrm{p}=0.044)$. The type of vascular procedure (surgical, intravascular, and hybrid) that had been performed before the amputation 
had no statistically significant influence on the time between the first vascular procedure and amputation. The multivariate analysis based on stepwise progressive regression resulted in an equation explaining $99 \%$ of the inter-procedure variations in duration of the leg-saving period. This analysis showed this parameter as being determined by a number of factors (Table 3).

Table 1. Comparison of clinical data of patients with primary amputation with patients who have had at least one revascularization procedure prior to the amputation (the analysis concerns 39 amputated legs

Tabela 1. Porównanie danych klinicznych pacjentów z pierwotna amputacja z chorymi, którzy przed amputacja kończyny mieli na niej wykonany przynajmniej jeden zabieg rewaskularyzacyjny (analiza w odniesieniu do zabiegu)

\begin{tabular}{|c|c|c|c|}
\hline Clinical characteristic & $\begin{array}{c}\text { Patients with } \\
\text { primary leg } \\
\text { amputation } \\
(\mathrm{n}=6 ; 15 \%)\end{array}$ & $\begin{array}{l}\text { Patients with } \\
\text { secondary } \\
\text { amputation } \\
(\mathbf{n}=\mathbf{3 3} ; \mathbf{8 5} \%)\end{array}$ & $\mathbf{p}$ \\
\hline Days hospitalized & $10.5 \pm 5.2$ & $10.2 \pm 6.7$ & 0.90 \\
\hline $\begin{array}{l}\text { Amputation of the left limb } \\
(\mathrm{n}, \%)\end{array}$ & $6(100 \%)$ & $19(58 \%)$ & 0.047 \\
\hline $\begin{array}{l}\text { Range (forefoot/calf/thigh) (n, } \\
\%)\end{array}$ & $\begin{array}{c}3(50 \%) / 1 \\
(17 \%) \\
2(33 \%)\end{array}$ & $\begin{array}{c}16(49 \%) / 5 \\
(15 \%) \\
12(36 \%)\end{array}$ & 0.98 \\
\hline $\begin{array}{l}\text { History of second leg } \\
\text { amputation }(\mathrm{n}, \%)\end{array}$ & $1(17 \%)$ & $8(24 \%)$ & 0.69 \\
\hline $\begin{array}{l}\text { Infection as a cause of } \\
\text { amputation }(n, \%)\end{array}$ & $4(67 \%)$ & $17(52 \%)$ & 0.75 \\
\hline Age (years) & $59.7 \pm 11.0$ & $67.8 \pm 9.4$ & 0.065 \\
\hline Male sex $(\mathrm{n}, \%)$ & $6(100 \%)$ & $23(70 \%)$ & 0.11 \\
\hline $\mathrm{CAD}(\mathrm{n}, \%)$ & $4(67 \%)$ & $14(42 \%)$ & 0.29 \\
\hline CABG history (n, \%) & $0(0 \%)$ & $6(18 \%)$ & 0.27 \\
\hline PCI history (n, \%) & $1(17 \%)$ & $4(12 \%)$ & 0.77 \\
\hline $\mathrm{CHF}(\mathrm{n}, \%)$ & $2(33 \%)$ & $11(33 \%)$ & 1.0 \\
\hline Atrial fibrillation (n, \%) & $2(33 \%)$ & $9(27 \%)$ & 0.77 \\
\hline Stroke history (n, \%) & $1(16 \%)$ & $5(15 \%)$ & 0.97 \\
\hline $\begin{array}{l}\text { Carotid artery stenosis } \\
(\mathrm{n}, \%)\end{array}$ & $0 \%$ & $5(15 \%)$ & 0.32 \\
\hline $\operatorname{COLD}(\mathrm{n}, \%)$ & $1(17 \%)$ & $3(9 \%)$ & 0.59 \\
\hline Tobacco smoking (n, \%) & $4(67 \%)$ & $18(56 \%)$ & 0.59 \\
\hline Arterial hypertension (n, \%) & $5(83 \%)$ & $30(91 \%)$ & 0.59 \\
\hline Diabetes mellitus (n, \%) & $4(67 \%)$ & $18(55 \%)$ & 0.93 \\
\hline Anemia (n, \%) & $5(83 \%)$ & $21(64 \%)$ & 0.36 \\
\hline CKD (n, \%) & $3(50 \%)$ & $9(27 \%)$ & 0.28 \\
\hline Dyslipidemia (n, \%) & $2(33 \%)$ & $22(67 \%)$ & 0.24 \\
\hline LDL cholesterol (mg/dl) & $98.8 \pm 23.9$ & $112.0 \pm 38.9$ & 0.48 \\
\hline Triglycerides (mg/dl) & $118.2 \pm 29.6$ & $122.4 \pm 46.0$ & 0.85 \\
\hline Glucose (mg/dl) & $177.3 \pm 51.6$ & $160.1 \pm 71.9$ & 0.58 \\
\hline
\end{tabular}

\begin{tabular}{|c|c|c|c|}
\hline Erythrocytes (T/1) & $3.6 \pm 0.6$ & $3.9 \pm 0.5$ & 0.14 \\
\hline Hemoglobin (g/dl) & $10.8 \pm 1.7$ & $11.4 \pm 1.9$ & 0.49 \\
\hline Hematocrit (\%) & $33.0 \pm 4.4$ & $34.5 \pm 5.3$ & 0.53 \\
\hline MCV (fl) & $92.5 \pm 6.1$ & $88.4 \pm 4.9$ & 0.075 \\
\hline $\mathrm{MCH}(\mathrm{pg})$ & $30.2 \pm 1.5$ & $29.0 \pm 1.7$ & 0.13 \\
\hline Leukocytes (G/l) & $12.4 \pm 6.0$ & $13.9 \pm 6.3$ & 0.57 \\
\hline CRP (mg/dl) & $110.9 \pm 56.6$ & $78.2 \pm 71.5$ & 0.34 \\
\hline Platelets (G/l) & $410.5 \pm 226.6$ & $346.3 \pm 124.9$ & 0.32 \\
\hline MPV (fl) & $8.6 \pm 1.2$ & $13.3 \pm 17.9$ & 0.53 \\
\hline INR & $1.15 \pm 0.098$ & $1.14 \pm 0.2$ & 0.89 \\
\hline aPTT (s) & $34.1 \pm 6.9$ & $32.0 \pm 5.7$ & 0.41 \\
\hline Creatinine (mg/dl) & $1.99 \pm 1.11$ & $1.37 \pm 0.80$ & 0.11 \\
\hline $\operatorname{GFR}(\mathrm{ml} / \mathrm{min})$ & $55.2 \pm 37.3$ & $76.5 \pm 29.5$ & 0.16 \\
\hline Aspirin therapy (n, \%) & $4(66 \%)$ & $32(97 \%)$ & 0.01 \\
\hline Clopidogrel therapy (n, \%) & $1(17 \%)$ & $23(70 \%)$ & 0.013 \\
\hline $\begin{array}{l}\text { Oral anticoagulant therapy } \\
(\mathrm{n}, \%)\end{array}$ & $2(33 \%)$ & $20(61 \%)$ & 0.23 \\
\hline Statin therapy $(\mathrm{n}, \%)$ & $3(50 \%)$ & $31(94 \%)$ & 0.002 \\
\hline ACEI therapy (n, \%) & $5(83 \%)$ & $26(79 \%)$ & 0.81 \\
\hline $\begin{array}{l}\text { Calcium channel blocker } \\
\text { therapy }(\mathrm{n}, \%)\end{array}$ & 0 & $7(21 \%)$ & 0.22 \\
\hline Beta-blocker therapy (n, \%) & $4(69 \%)$ & $23(70 \%)$ & 0.89 \\
\hline
\end{tabular}

Abbreviations: $\mathrm{CAD}=$ coronary artery disease; $\mathrm{CABG}=$ coronary artery bypass graft; $\mathrm{PCI}=$ percutaneous coronary intervention; $\mathrm{CHF}$ $=$ coronary heart failure; $\mathrm{COLD}=$ chronic obstructive lung disease; $\mathrm{CKD}=$ chronic kidney disease; $\mathrm{LDL}=$ low-density lipoprotein; $\mathrm{MCV}=$ mean corpuscular volume; $\mathrm{MCH}=$ mean corpuscular hemoglobin; $\mathrm{CRP}=\mathrm{C}$-reactive protein; $\mathrm{MPV}=$ mean platelet volume; INR = international normalized ratio; $\mathrm{aPTT}=$ activated partial thromboplastin time; GFR $=$ glomerular filtration rate; ACEI $=$ angiotensin converting enzyme inhibitor

Table 2. Comparison of the characteristics of patients with an amputation performed at the thigh level with patients who underwent amputation at the forefoot level (analysis per procedure)

Tabela 2. Porównanie charakterystyki pacjentów z amputacja wykonana na udzie $w$ porównaniu z chorymi, $u$ których wykonano amputacje na poziomie przodostopia

\begin{tabular}{|l|c|c|c|}
\hline $\begin{array}{l}\text { Clinical characteristic } \\
\text { Cecha kliniczna }\end{array}$ & $\begin{array}{c}\text { Patients with } \\
\text { thigh amputation } \\
\text { (n= 14) } \\
\text { Pacjenci z } \\
\text { amputacją na } \\
\text { udzie } \\
(\mathrm{n}=14)\end{array}$ & $\begin{array}{c}\text { Patients } \\
\text { with } \\
\text { forefoot } \\
\text { amputation } \\
\text { (n= 19) } \\
\text { Pacjenci } \\
\text { z amputacją } \\
\text { przodosto- } \\
\text { pia (n=21) }\end{array}$ & $\mathrm{p}$ \\
\hline $\begin{array}{l}\text { Days hospitalized due to } \\
\text { amputation }\end{array}$ & $15.14 \pm 6.5$ & $7.21 \pm 5.2$ & 0.0005 \\
$\begin{array}{l}\text { Dni hospitalizacji związanej } \\
\text { z amputacją }\end{array}$ & $148.6 \pm 248.9$ & $192.5 \pm$ & 0.66 \\
\hline $\begin{array}{l}\text { Length of time between } \\
\text { procedure and amputation } \\
\text { (days) } \\
\text { czas między zabiegiem a } \\
\text { amputacją (dni) }\end{array}$ & & & \\
\hline
\end{tabular}


Characteristics of patients who underwent lower limb amputation due to critical limb ischemia - one center experience

\begin{tabular}{|c|c|c|c|}
\hline $\begin{array}{l}\text { Left limb amputation (n, \%) } \\
\text { Amputacja lewej kończyny (n, } \\
\%)\end{array}$ & $10(71 \%)$ & $13(68 \%)$ & 0.86 \\
\hline $\begin{array}{l}\text { History of second leg } \\
\text { amputation (n, \%) } \\
\text { Amputacja drugiej kończyny } \\
\text { dolnej w wywiadzie (n, \%) }\end{array}$ & $4(29 \%)$ & $3(16 \%)$ & 0.27 \\
\hline $\begin{array}{l}\text { Age (years) } \\
\text { Wiek (lata) }\end{array}$ & $69.3 \pm 7.2$ & $62.2 \pm 9.5$ & 0.026 \\
\hline $\begin{array}{l}\text { Male sex (n, \%) } \\
\text { płeć męska (n, \%) }\end{array}$ & $11(79 \%)$ & $14(74 \%)$ & 0.76 \\
\hline $\begin{array}{l}\text { CAD (n, \%) } \\
\text { Choroba niedokrwienna serca } \\
(\mathrm{n}, \%)\end{array}$ & $9(64 \%)$ & $7(37 \%)$ & 0.13 \\
\hline $\begin{array}{l}\text { CABG history }(\mathrm{n}, \%) \\
\text { CABG w wywiadzie }(\mathrm{n}, \%)\end{array}$ & $1(7 \%)$ & $4(21 \%)$ & 0.29 \\
\hline $\begin{array}{l}\text { PCI history }(\mathrm{n}, \%) \\
\text { PCI wieńcowe w wywiadzie } \\
(\mathrm{n}, \%)\end{array}$ & $2(14 \%)$ & $1(5 \%)$ & 0.39 \\
\hline $\begin{array}{l}\text { CHF }(\mathrm{n}, \%) \\
\text { Przewlekła niewydolność serca } \\
(\mathrm{n}, \%)\end{array}$ & $7(50 \%)$ & $3(16 \%)$ & 0.035 \\
\hline $\begin{array}{l}\text { *Atrial fibrillation (n, \%) } \\
\text { Migotanie przedsionków } \\
(\mathrm{n}, \%)\end{array}$ & $6(43 \%)$ & $5(26 \%)$ & 0.33 \\
\hline $\begin{array}{l}\text { Stroke history (n, \%) } \\
\text { Przebyty udar (n, \%) }\end{array}$ & $5(36 \%)$ & $1(5 \%)$ & 0.029 \\
\hline $\begin{array}{l}\text { Carotid artery stenosis (n, \%) } \\
\text { Zwężenie tętnicy szyjnej (n, } \\
\% \text { ) }\end{array}$ & $2(14 \%)$ & $2(10 \%)$ & 0.75 \\
\hline $\begin{array}{l}\operatorname{COLD}(\mathrm{n}, \%) \\
\operatorname{POCHP}(\mathrm{n}, \%)\end{array}$ & $1(7 \%)$ & $2(10 \%)$ & 0.75 \\
\hline $\begin{array}{l}\text { Tobacco smoking }(\mathrm{n}, \%) \\
\text { Palenie }(\mathrm{n}, \%)\end{array}$ & $6(43 \%)$ & $14(74 \%)$ & 0.077 \\
\hline $\begin{array}{l}\text { Arterial hypertension (n, \%) } \\
\text { Nadciśnienie tętnicze (n, \%) }\end{array}$ & $13(93 \%)$ & $17(89 \%)$ & 0.75 \\
\hline $\begin{array}{l}\text { Diabetes mellitus (n, \%) } \\
\text { Cukrzyca (n, \%) }\end{array}$ & $8(57 \%)$ & $11(58 \%)$ & 0.86 \\
\hline $\begin{array}{l}\text { Anemia (n, \%) } \\
\text { Niedokrwistość (n, \%) }\end{array}$ & $12(86 \%)$ & $11(58 \%)$ & 0.091 \\
\hline $\begin{array}{l}\text { CKD (n, \%) } \\
\text { Niewydolność nerek (n, \%) }\end{array}$ & $8(57 \%)$ & $2(10 \%)$ & 0.003 \\
\hline $\begin{array}{l}\text { Dyslipidemia (n, \%) } \\
\text { Dyslipidemia (n, \%) }\end{array}$ & $10(71 \%)$ & $10(48 \%)$ & 0.83 \\
\hline $\begin{array}{l}\text { LDL cholesterol (mg/dl) } \\
\text { Cholesterol LDL (mg/dl) }\end{array}$ & $109.5 \pm 37.1$ & $\begin{array}{c}114.9 \pm \\
40.2\end{array}$ & 0.78 \\
\hline $\begin{array}{l}\text { Triglycerides (mg/dl) } \\
\text { Triglicerydy (mg/dl) }\end{array}$ & $105.4 \pm 29.8$ & $\begin{array}{c}133.2 \pm \\
49.7\end{array}$ & 0.2 \\
\hline $\begin{array}{l}\text { Glucose (mg/dl) } \\
\text { Glukoza (mg/dl) }\end{array}$ & $155.9 \pm 45.1$ & $\begin{array}{c}159.8 \pm \\
84.7\end{array}$ & 0.88 \\
\hline $\begin{array}{l}\text { Erythrocytes (T/l) } \\
\text { Erytrocyty }(\mathrm{T} / \mathrm{l})\end{array}$ & $3.8 \pm 0.6$ & $4.0 \pm 0.5$ & 0.34 \\
\hline $\begin{array}{l}\text { Hemoglobin }(\mathrm{g} / \mathrm{dl}) \\
\text { Hemoglobina }(\mathrm{g} / \mathrm{dl})\end{array}$ & $10.9 \pm 2.0$ & $11.7 \pm 1.9$ & 0.25 \\
\hline $\begin{array}{l}\text { Hematocrit (\%) } \\
\text { Hematokryt (\%) }\end{array}$ & $33.4 \pm 5.4$ & $35.2 \pm 5.4$ & 0.35 \\
\hline $\begin{array}{l}\text { MCV (fl) } \\
\text { MCV (fl) }\end{array}$ & $89.2 \pm 7.3$ & $88.9 \pm 3.7$ & 0.92 \\
\hline $\begin{array}{l}\mathrm{MCH}(\mathrm{pg}) \\
\mathrm{MCH}(\mathrm{pg})\end{array}$ & $29.0 \pm 2.1$ & $29.3 \pm 1.5$ & 0.62 \\
\hline Leukocyte blood count (G/l) & $15.7 \pm 8.2$ & $12.2 \pm 4.8$ & 0.13 \\
\hline
\end{tabular}

\begin{tabular}{|c|c|c|c|}
\hline Leukocyty (G/l) & & & \\
\hline $\begin{array}{l}\text { CRP (mg/dl) } \\
\text { CRP (mg/dl) }\end{array}$ & $88.2 \pm 59.7$ & $56.9 \pm 59.9$ & 0.19 \\
\hline $\begin{array}{l}\text { Platelet count (G/l) } \\
\text { Płytki krwi }(\mathrm{G} / \mathrm{l})\end{array}$ & $318.1 \pm 27.8$ & $\begin{array}{c}375.2 \pm \\
155.3\end{array}$ & 0.27 \\
\hline $\begin{array}{l}\text { MPV (fl) } \\
\text { MPV (fl) }\end{array}$ & $19.6 \pm 26.3$ & $8.4 \pm 0.9$ & 0.08 \\
\hline $\begin{array}{l}\text { INR } \\
\text { INR }\end{array}$ & $1.2 \pm 0.2$ & $1.1 \pm 0.2$ & 0.32 \\
\hline $\begin{array}{l}\text { aPTT (s) } \\
\text { aPTT (s) }\end{array}$ & $34.5 \pm 7.3$ & $31.2 \pm 4.2$ & 0.11 \\
\hline $\begin{array}{l}\text { Creatinine }(\mathrm{mg} / \mathrm{dl}) \\
\text { Kreatynina }(\mathrm{mg} / \mathrm{dl})\end{array}$ & $2.0 \pm 1.2$ & $1.13 \pm 0.2$ & 0.005 \\
\hline $\begin{array}{l}\text { GFR (ml/min) } \\
\text { GFR (ml/min) }\end{array}$ & $62.9 \pm 36.3$ & $82.0 \pm 24.7$ & 0.086 \\
\hline $\begin{array}{l}\text { Aspirin therapy (n, \%) } \\
\text { Leczenie aspiryną (n, \%) }\end{array}$ & $14(100 \%)$ & $16(84 \%)$ & 0.13 \\
\hline $\begin{array}{l}\text { Clopidogrel therapy }(\mathrm{n}, \%) \\
\text { Leczenie klopidogrelem } \\
(\mathrm{n}, \%)\end{array}$ & $11(79 \%)$ & $9(47 \%)$ & 0.07 \\
\hline $\begin{array}{l}\text { Oral anticoagulant therapy (n, } \\
\%) \\
\text { Doustny antykoagulant (n, \%) }\end{array}$ & $9(64 \%)$ & $8(42 \%)$ & 0.22 \\
\hline $\begin{array}{l}\text { Statin therapy }(\mathrm{n}, \%) \\
\text { Leczenie statyną (n, \%) }\end{array}$ & $12(85 \%)$ & $16(84 \%)$ & 0.91 \\
\hline $\begin{array}{l}\text { ACEI therapy (n, \%) } \\
\text { Leczenie ACEI }(n, \%)\end{array}$ & $10(71 \%)$ & $16(84 \%)$ & 0.39 \\
\hline $\begin{array}{l}\text { Calcium channel blocker } \\
\text { therapy }(\mathrm{n}, \%) \\
\text { Leczenie blokerem kanałów } \\
\text { wapniowych }(\mathrm{n}, \%)\end{array}$ & $3(21 \%)$ & $4(21 \%)$ & 0.98 \\
\hline $\begin{array}{l}\text { Beta-blocker therapy (n, \%) } \\
\text { Leczenie beta-blokerem (n, \%) }\end{array}$ & $12(86 \%)$ & $11(58 \%)$ & 0.091 \\
\hline $\begin{array}{l}\text { Endovascular procedure } \\
\text { history }(\mathrm{n}, \%) \\
\text { Wcześniejsze zabiegi } \\
\text { wewnątrznaczyniowe (n, \%) }\end{array}$ & $12(86 \%)$ & $15(79 \%)$ & 0.63 \\
\hline $\begin{array}{l}\text { Severity of changes at } \\
\text { aortoiliac level according to } \\
\text { TASC II }(0 / \mathrm{A} / \mathrm{B} / \mathrm{C} / \mathrm{D}) \\
\text { Zaawansowanie zmian } \\
\text { w odcinku aortalno- } \\
\text { biodrowym wg TASC II } \\
\text { (0/A/B/C/D) }\end{array}$ & $\begin{array}{c}7(50 \%) / 1(7 \%) / \\
1(7 \%) / 2(14 \%) / \\
3(22 \%)\end{array}$ & $\begin{array}{l}10(48 \%) / \\
1(4 \%) / \\
2(10 \%) / \\
4(19 \%) / \\
4(19 \%)\end{array}$ & 0.12 \\
\hline $\begin{array}{l}\text { Severity of changes at } \\
\text { femoropopliteal level } \\
\text { according to TASC II } \\
(0 / \mathrm{A} / \mathrm{B} / \mathrm{C} / \mathrm{D}) \\
\text { Zaawansowanie zmian w } \\
\text { odcinku udowo- } \\
\text { podkolanowym wg TASC II } \\
(0 / \mathrm{A} / \mathrm{B} / \mathrm{C} / \mathrm{D})\end{array}$ & $\begin{array}{c}3(22 \%) / 4(38 \%) / \\
2(14 \%) / 2(14 \%) / \\
3(22 \%)\end{array}$ & $\begin{array}{l}5(24 \%) / \\
2(10 \%) / \\
4(19 \%) / \\
3(14 \%) / \\
7(33 \%)\end{array}$ & 0.66 \\
\hline $\begin{array}{l}\text { Number of permeable arteries } \\
\text { in lower leg } \\
\text { Liczba drożnych tętnic goleni }\end{array}$ & $2.2 \pm 1.0$ & $1.9 \pm 1.0$ & 0.49 \\
\hline $\begin{array}{l}\text { Number of permeable arteries } \\
\text { in foot } \\
\text { Liczba drożnych tętnic stopy }\end{array}$ & $1.3 \pm 0.7$ & $1.1 \pm 0.9$ & 0.58 \\
\hline $\begin{array}{l}\text { Level of the first } \\
\text { revascularization ( } 0 \text { / aortoiliac } \\
\text { / femoropopliteal / below the } \\
\text { knee / at least two segments) } \\
\text { Poziom pierwszego zabiegu } \\
\text { (0/aortalno-biodrowy/ udowo- } \\
\text { podkolanowy/ poniżej kolana, } \\
\text { przynajmniej } 2 \text { segmenty) }\end{array}$ & $\begin{array}{c}2(14 \%) / 3(22 \%) / \\
2(14 \%) / 2(14 \%) / \\
5(36 \%)\end{array}$ & $\begin{array}{l}4(19 \%) / \\
2(9 \%) / \\
3(14 \%) / \\
6(29 \%) / \\
6(29 \%)\end{array}$ & 0.51 \\
\hline $\begin{array}{l}\text { Occlusion of treated artery } \\
(\mathrm{n}, \%)\end{array}$ & $7(50 \%)$ & $12(57 \%)$ & 0.13 \\
\hline
\end{tabular}


Characteristics of patients who underwent lower limb amputation due to critical limb ischemia - one center experience

\begin{tabular}{|c|c|c|c|}
\hline $\begin{array}{l}\text { Niedrożność tętnicy poddanej } \\
\text { zabiegowi }(\mathrm{n}, \%)\end{array}$ & & & \\
\hline $\begin{array}{l}\text { The number of stents } \\
\text { implanted during a previous } \\
\text { surgery (n) } \\
\text { Liczba stentów } \\
\text { implantowanych podczas } \\
\text { wcześniejszego zabiegu (n) }\end{array}$ & $1.0 \pm 1.0$ & $1.3 \pm 1.4$ & 0.59 \\
\hline $\begin{array}{l}\text { Total length of the stents }(\mathrm{mm}) \\
\text { Suma długości stentów }(\mathrm{mm})\end{array}$ & $87.6 \pm 46.3$ & $110.5 \pm 7.0$ & 0.19 \\
\hline $\begin{array}{l}\text { Implantation of DES (n, \%) } \\
\text { Implantacja DES do tętnicy } \\
(\mathrm{n}, \%)\end{array}$ & $8(57 \%)$ & $8(39 \%)$ & 0.71 \\
\hline $\begin{array}{l}\text { Use of DEB (n, \%) } \\
\text { Zastosowanie DEB (n, \%) }\end{array}$ & $12(86 \%)$ & $15(71 \%)$ & 0.47 \\
\hline $\begin{array}{l}\text { Implantation of a stent into the } \\
\text { popliteal artery during previous } \\
\text { surgery (n, \%) } \\
\text { Implantacja stentu do tętnicy } \\
\text { podkolanowej podczas } \\
\text { wcześniejszego zabiegu (n, \%) }\end{array}$ & $3(21 \%)$ & $4(19 \%)$ & 1.00 \\
\hline $\begin{array}{l}\text { TLR before amputation (n, \%) } \\
\text { Liczba zabiegów naprawczych } \\
\text { (TLR) przed amputacją (n, \%) }\end{array}$ & $10(71 \%)$ & $12(57 \%)$ & 0.31 \\
\hline
\end{tabular}

Abbreviations: $\mathrm{CAD}=$ coronary artery disease; $\mathrm{CABG}=$ coronary artery bypass graft; $\mathrm{PCI}=$ percutaneous coronary intervention; $\mathrm{CHF}$ $=$ coronary heart failure COLD $=$ chronic obstructive lung disease; $\mathrm{CKD}=$ chronic kidney disease $; \mathrm{LDL}=$ low-density lipoprotein; $\mathrm{MCV}=$ mean corpuscular volume; $\mathrm{MCH}=$ mean corpuscular hemoglobin; CRP = C-reactive protein; $\mathrm{MPV}=$ mean platelet volume; INR = international normalized ratio; aPTT = activated partial thromboplastin time; GFR = glomerular filtration rate; ACEI $=$ angiotensin converting enzyme inhibitor; DES = drug eluting stent; $\mathrm{DEB}=$ drug eluting balloon; TLR = target lesion revascularization

Table 3. Results of the multivariate analysis based on progressive stepwise regression for the length of time between the first revascularization procedure and a secondary amputation in one leg. $R=0.99 ; R^{2}=0.99 ;$ corrected $R^{2}=0.99 ; F$ $(32.1)=78292 ; p<0.0028 ;$ estimated standard error $=0.99$

Tabela 3. Wyniki krokowo-postępującej regresji wielokrotnej dla czasu trwania okresu między pierwszym zabiegiem rewaskularyzacyjnym a wykonaniem amputacji leczonej wcześniej kończyny. $R=0,99$; $R^{2}=0,99 ;$ popraw. $R^{2}=0,99 ; F(31,1)=78292 ; p<$ 0,0028; błąd stand. estymacji: 0,985

\begin{tabular}{|l|c|c|c|c|c|c|}
\hline $\begin{array}{l}\text { Parameter } \\
\text { Parametr }\end{array}$ & $\boldsymbol{\beta}$ & $\begin{array}{c}\boldsymbol{\beta} \\
\text { stand. } \\
\text { error }\end{array}$ & $\mathbf{b}$ & $\begin{array}{c}\text { b } \\
\text { stand. } \\
\text { error }\end{array}$ & T(1) & $\mathbf{p}$ \\
\hline $\begin{array}{l}\text { Constant } \\
\text { Wyraz wolny }\end{array}$ & -2.38 & 0.02 & -376.9 & 3.4 & - & 0.011 .3 \\
\hline $\begin{array}{l}\text { MCH (pg) } \\
\text { MCH (pg) }\end{array}$ & 0.18 & 0.002 & 41.0 & 0.6 & 68.8 & 0.01 \\
\hline $\begin{array}{l}\text { Number of } \\
\text { revascularization } \\
\text { procedures } \\
\text { liczba zabiegów } \\
\text { rewaskularyzacyjnych }\end{array}$ & 2.99 & 0.02 & 167.2 & 1.0 & 165.2 & 0.004 \\
\hline $\begin{array}{l}\text { MCV (fl) } \\
\text { MCV (fl) }\end{array}$ & 0.32 & 0.01 & 13.6 & 0.3 & 41.7 & 0.02 \\
\hline $\begin{array}{l}\text { Leukocytes (G/l) } \\
\text { Liczba leukocytów } \\
\text { (G/l) }\end{array}$ & & & & & & 0.01 \\
\hline
\end{tabular}

\begin{tabular}{|c|c|c|c|c|c|c|}
\hline $\begin{array}{l}\text { INR } \\
\text { INR }\end{array}$ & -1.33 & 0.01 & -1656.1 & 5.8 & $\begin{array}{c}- \\
286.3\end{array}$ & 0.002 \\
\hline $\begin{array}{l}\text { Tobacco smoking } \\
\text { habit } \\
\text { Palenie tytoniu }\end{array}$ & -0.93 & 0.01 & -496.9 & 4.6 & $\begin{array}{c}- \\
108.8\end{array}$ & 0.006 \\
\hline $\begin{array}{l}\text { MPV (fl) } \\
\text { MPV (fl) }\end{array}$ & -0.01 & 0.01 & -0.1 & 0.1 & -1.5 & 0.37 \\
\hline $\begin{array}{l}\text { Atrial fibrillation } \\
\text { Migotanie } \\
\text { przedsionków }\end{array}$ & 0.67 & 0.004 & 404.3 & 2.3 & 173.4 & 0.004 \\
\hline $\begin{array}{l}\text { Total length of stents } \\
\text { Suma długości } \\
\text { implantowanych } \\
\text { stentów }\end{array}$ & -0.42 & 0.003 & -0.8 & 0.0063 & ${ }^{-}$ & 0.005 \\
\hline $\begin{array}{l}\text { Occlusion of treated } \\
\text { artery } \\
\text { Niedrożność } \\
\text { rewaskularyzowanej } \\
\text { tętnicy }\end{array}$ & 0.01 & 0.008 & 6.5 & 4.2 & 1.5 & 0.37 \\
\hline $\begin{array}{l}\text { Number of implanted } \\
\text { stents } \\
\text { liczba } \\
\text { implantowanych } \\
\text { stentów }\end{array}$ & 0.84 & 0.01 & 202.1 & 3.4 & 59.1 & 0.01 \\
\hline $\begin{array}{l}\text { Hemoglobin }(\mathrm{g} / \mathrm{dl}) \\
\text { HB }(\mathrm{g} / \mathrm{dl})\end{array}$ & 5.42 & 0.05 & 785.4 & 6.9 & 112.7 & 0.006 \\
\hline $\begin{array}{l}\text { Hematocrit (\%) } \\
\text { HT }(\%)\end{array}$ & -5.60 & 0.04 & -285.7 & 2.3 & 125.7 & 0.005 \\
\hline $\begin{array}{l}\text { Age (years) } \\
\text { Wiek (lata) }\end{array}$ & -0.77 & 0.004 & -22.3 & 0.1 & 184.6 & 0.003 \\
\hline $\begin{array}{l}\text { Rutherford (class 4-6) } \\
\text { Rutherford (klasa 4-6) }\end{array}$ & -0.21 & 0.01 & -91.2 & 2.1 & -44.3 & 0.01 \\
\hline $\begin{array}{l}\text { CABG history } \\
\text { CABG w wywiadzie }\end{array}$ & -0.24 & 0.01 & -163.3 & 3.9 & -40.9 & 0.02 \\
\hline $\begin{array}{l}\text { PCI history } \\
\text { PTCA w wywiadzie }\end{array}$ & -0.84 & 0.003 & -684.7 & 2.6 & $\begin{array}{c}- \\
258.7\end{array}$ & 0.002 \\
\hline $\begin{array}{l}\text { Diabetes mellitus } \\
\text { Cukrzyca }\end{array}$ & 0.04 & 0.01 & 22.8 & 3.2 & 7.2 & 0.09 \\
\hline $\begin{array}{l}\text { Glucose }(\mathrm{mg} / \mathrm{dl}) \\
\text { Glukoza we krwi } \\
(\mathrm{mg} / \mathrm{dl})\end{array}$ & 0.14 & 0.01 & 0.6 & 0.04 & 14.1 & 0.04 \\
\hline $\begin{array}{l}\text { CAD history } \\
\text { Choroba } \\
\text { niedokrwienna serca } \\
\text { w wywiadzie }\end{array}$ & -0.03 & 0.01 & -18.0 & 5.1 & -3.5 & 0.18 \\
\hline $\begin{array}{l}\text { Dyslipidemia } \\
\text { Dyslipidemia w } \\
\text { wywiadzie }\end{array}$ & -0.30 & 0.01 & -162.3 & 3.0 & -54.2 & 0.012 \\
\hline $\begin{array}{l}\text { Severity of changes at } \\
\text { femoropopliteal level } \\
\text { according to TASC II } \\
\text { Nasilenie zmian w } \\
\text { odcinku udowo- } \\
\text { podkolanowym wg } \\
\text { TASC-II }\end{array}$ & -0.1 & 0.01 & -0.6 & 0.05 & -12.5 & 0.05 \\
\hline $\begin{array}{l}\text { aPTT (s) } \\
\text { aPTT (s) }\end{array}$ & 0.31 & 0.003 & 15.3 & 0.1 & 123.9 & 0.005 \\
\hline $\begin{array}{l}\text { Severity of changes at } \\
\text { aortoiliac level } \\
\text { according to TASC II } \\
\text { Nasilenie zmian w } \\
\text { odcinku aortalno- } \\
\text { biodrowym wg } \\
\text { TASC-II }\end{array}$ & 0.44 & 0.002 & 2.5 & 0.01 & 178.4 & 0.002 \\
\hline $\begin{array}{l}\text { Platelet count (G/l) } \\
\text { Liczba płytek krwi } \\
\text { (G/l) }\end{array}$ & 0.23 & 0.01 & 0.5 & 0.02 & 32.4 & 0.02 \\
\hline Statin therapy & 0.19 & 0.003 & 207.6 & 3.1 & 68.1 & 0.01 \\
\hline
\end{tabular}




\begin{tabular}{|c|c|c|c|c|c|c|}
\hline Leczenie statyną & & & & & & \\
\hline $\begin{array}{l}\text { Severity of changes in } \\
\text { previously treated } \\
\text { segment according to } \\
\text { TASC-II } \\
\text { Nasilenie z zmian w } \\
\text { leczonym wcześniej } \\
\text { odcinku wg TASC }\end{array}$ & 0.69 & 0.02 & 94.6 & 2.3 & 41.8 & 0.02 \\
\hline $\begin{array}{l}\text { Stroke history } \\
\text { UDAR w wywiadzie }\end{array}$ & 0.39 & 0.01 & 288.0 & 9.6 & 29.9 & 0.02 \\
\hline $\begin{array}{l}\text { Cholesterol LDL } \\
\text { (mg/dl) } \\
\text { Stężenie LDL (mg/dl) }\end{array}$ & 0.11 & 0.003 & 1.0 & 0.03 & 35.2 & 0.02 \\
\hline $\begin{array}{l}\text { Gender (male / } \\
\text { female) } \\
\text { Płeć (męska/ żeńska) }\end{array}$ & 0.06 & 0.003 & 32.2 & 1.9 & 16.2 & 0.04 \\
\hline $\begin{array}{l}\text { Creatinine }(\mathrm{mg} / \mathrm{dl}) \\
\text { Stężenie kreatyniny } \\
(\mathrm{mg} / \mathrm{dl})\end{array}$ & 0.01 & 0.005 & 4.8 & 1.6 & 2.9 & 0.21 \\
\hline
\end{tabular}

Abbreviations: $\mathrm{MCH}=$ mean corpuscular hemoglobin; $\mathrm{MCV}=$ mean corpuscular volume; INR = international normalized ratio; $\mathrm{MPV}=$ mean platelet volume $\mathrm{CABG}=$ coronary artery bypass graft; $\mathrm{PCI}=$ percutaneous coronary intervention; $\mathrm{CAD}=$ coronary artery disease; aPTT $=$ activated partial thromboplastin time; $\mathrm{LDL}=$ lowdensity lipoprotein

\section{DISCUSSION}

The study retrospectively analyzed risk factors for the amputation of 39 lower limbs performed in our clinic between 2012 and 2014. The variables determining the duration of the period between the first revascularization and secondary amputation in multifactorial analysis were also defined. The only significant risk factors for primary amputation were the absence of aspirin, clopidogrel and statin pharmacotherapy (Table 1), which was most likely due to a delay in diagnosing the chronic lower limb ischemia. Older age, CHF and CKD were associated with an increased probability of a major amputation at the thigh level (Table 2). Multivariate analysis showed that the length of time between the first revascularization procedure and limb amputation was determined by a number of factors (Table 3), such as: the severity of atherosclerosis in the arteries of the aortoiliac segment (TransAtlantic Intersociety Consensus II; TASC II) [3], the presence of risk factors for atherosclerosis (e.g. tobacco smoking), use of adequate pharmacotherapy (e.g. statins), cardiovascular comorbidity (e.g. atrial fibrillation or a history of stroke), as well as some abnormalities in hematological or biochemistry laboratory values (e.g. leukocyte count, platelet count, INR, activated partial thromboplastin time [aPTT]). These data corroborate the results of other publications [3]. However, it is puzzling that diabetes mellitus, which is recognized as one of the strongest risk factors for CLI and lower limb

amputation [3], showed similar prevalence in patients with primary and secondary amputation (Table 1), as well as with amputation at the thigh and forefoot level (Table 2). This factor was also not included in the multiple regression model determining the length of time between the first revascularization and amputation (Table 3). These discrepancies were most likely to have resulted from the relatively small number of patients studied or from better diabetes care than ten years ago, when TASC II was prepared. On the other hand, a smoking habit, atrial fibrillation and age achieved statistical significance (Table 3). Another interesting and seemingly paradoxical finding was the positive correlation of LDL cholesterol level with the length of time between the first procedure and amputation (Table 3), indicating that higher LDL cholesterol level delayed the amputation and suggesting a leg-saving effect, similar to the lipidlowering function of statins (Table 3), and in contrast with widely accepted data showing on harmful effect of dyslipidemia [4-9] and the favorable effect of statin therapy [7, 10-12]. The only reasonable explanation of these discrepancies is a lipid-lowering effect of cachexia reported previously in patients with CLI [13]. In this context, a low LDL cholesterol level did not reflect the level of the risk factor of atherosclerosis, but only the degree of metabolic devastation of the organism. Similar conclusions were drawn by Owens et al. [13] among patients who were qualified for a femoropopliteal bypass. In that cohort, those with CLI who died during the observation period had a significantly lower LDL cholesterol concentration than patients with intermittent claudication who had survived the observation period. Owens et al. [13] indicated a positive correlation between blood cholesterol and albumin concentration, which, in their opinion, confirmed an association between limb amputation and a patient's poor nutritional status. In this regard, our observation does not contradict the recognition of dyslipidemia as a factor of risk and progression [3-7]. In contrast, the positive effect of statin intake on delaying amputation (Table 3) and the lower prevalence of statin therapy in the group of patients with a primary amputation (Table 1) suggest that the effect of pleiotropic statins is predominant in relation to the lipid-lowering effect in the prevention of amputation. Many data confirm the positive impact of statins on the course of lower limb ischemia [10-12, 14-16], claudication distance [10, 11, 17], and the prevalence of cardiovascular events in patients with 
CLI [15, 18, 19], although statin therapy has no influence on the risk of amputation [16].

The positive correlation of the severity of changes in the aortoiliac segment according to TASC II and its update $[3,20]$ and the length of time between the first procedure and amputation (Table 3 ) also seems to be paradoxical. However, this should come as no surprise when taking into consideration the fact that in medicine the sickest receive the greatest benefit. For example, patients who undergo coronary revascularization for cardiogenic shock or the efficacy of thrombolysis for stroke. Furthermore, the greater severity of the atherosclerotic changes in the proximal arteries of the lower limb, the stronger the release of angiogenesis stimulating factors [21], which can improve the peripheral perfusion.

There are some practical implications resulting from this study. Firstly, it is worth considering a screening program for lower limb ischemia, at least in certain groups of patients, to prevent delays both in the diagnosis of PAD and in introducing treatment with aspirin and statins, which improve patients' symptoms, improve the outcome of leg revascularization and can prolong the time between a procedure and amputation (Tables 1 and 3). Secondly, in our study, only $15 \%$ of patients underwent primary leg amputation, which is a better result than previously reported $50 \%$ of primary amputations [3]. Thirdly, we found a positive correlation between the number of revascularizations and delayed amputation time (Table 3). Therefore, every patient with CLI should, if possible, undergo lower limb revascularization and, in the case of the recurrence of leg ischemia (e.g. due to stent insufficiency), it is always worth performing another procedure with stent implantation with the required number of stents (Table 3). As a result, controversies concerning extensive stenting of lower extremity arteries and a recommendation to avoid "full metal jacket" stenting seem to appeal more to patients with intermittent claudication than with CLI [21-23]. Fourthly, statin therapy for patients with PAD should be recommended not only for atherosclerosis of lower limb arteries, but also for cardiovascular comorbidities, such as coronary artery diseases and previous strokes (Tables 1 and 2). The treatment should aim to achieve the target level of blood lipids recommended by the European Society of Cardiology [4-7].

The authors of this study are aware of its limitations. The low number of amputated limbs in the analysis and the disproportionate number of primary and secondary amputations may affect the strength and reliability of the reasoning. Despite these limitations, many of the comparisons made in the analyzed groups were found by the authors to be statistically significant.

\section{CONCLUSIONS}

1. Among patients who underwent lower limb amputation due to CLI, $15 \%$ were admitted to hospital in a condition that did not allow the performance of a revascularization procedure, which suggests the need for an improvement in patient education so that they can be referred earlier to vascular centers. A substantial percent (54\%) of the patients needed just a small amputation at the level of forefoot and did not need any further radical surgeries during the two years of observation, most probably due to the effect of previous revascularization procedures.

2. Patients who underwent a thigh-level amputation stayed in hospital twice as long compared with those who had less radical surgeries. The main factors determining the time between a revascularization procedure and amputation, as well as its level, were as follows: the number of procedures and stent implantations in the patient's history, the coexistence of CHF and CKD, tobacco smoking, inadequate pharmacotherapy, and the severity of changes revealed by angiography. Paradoxically, there was no influence of diabetes mellitus and dyslipidemia on these outcomes.

3. Amputations were delayed by successive revascularization procedures (at least two procedures in the majority of patients) and stenting the vessels. Therefore, it is worth to perform a revascularization procedure in every possible case, as well as it is necessary to inform patients to contact a vascular specialist without delay when symptoms of leg ischemia progression occur.

\section{REFERENCES}

1. Vemulapalli S, Greiner MA, Jones WS, et al. Peripheral arterial testing before lower extremity amputation among Medicare beneficiaries, 2000 to 2010. Circ Cardiovasc Qual Outcomes. 2014;7(1):142-50. doi: 10.1161/CIRCOUTCOMES.113.000376.

2. Yost ML. The cost of amputation in 2014. Lecture presented at the 4th annual Amputation Prevention Symposium. August 14-16, 2014 Palmer House Hilton, Chicago IL. 
3. Norgren L, Hiatt WR, Dormandy JA, et al. Inter-Society Consensus for the Management of Peripheral Arterial Disease (TASC II). Eur J Vasc Endovasc Surg. 2007;33 Suppl 1:S1-75.

4. European Stroke Organisation, Tendera M, Aboyans V et al. ESC Committee for Practice Guidelines. ESC Guidelines on the diagnosis and treatment of peripheral artery diseases: document covering atherosclerotic disease of extracranial carotid and vertebral, mesenteric, renal, upper and lower extremity arteries: the Task Force on the Diagnosis and Treatment of Peripheral Artery Diseases of the European Society of Cardiology (ESC). Eur Heart J. 2011;32(22):2851-906. doi: 10.1093/eurheartj/ehr211.

5. Ray KK, Kastelein JJ, Boekholdt SM et al. The ACC/AHA 2013 guideline on the treatment of blood cholesterol to reduce atherosclerotic cardiovascular disease risk in adults: the good the bad and the uncertain: a comparison with ESC/EAS guidelines for the management of dyslipidaemias 2011. Eur Heart J. 2014;35(15):960-8. doi: 10.1093/eurheartj/ehu107.

6. Perk J, De Backer G, Gohlke $\mathrm{H}$ et al. European Association for Cardiovascular Prevention \& Rehabilitation (EACPR); ESC Committee for Practice Guidelines (CPG). European Guidelines on cardiovascular disease prevention in clinical practice (version 2012). The Fifth Joint Task Force of the European Society of Cardiology and Other Societies on Cardiovascular Disease Prevention in Clinical Practice (constituted by representatives of nine societies and by invited experts). Eur Heart J. 2012;33(13):1635-701. doi: 10.1093/eurheartj/ehs092.

7. European Association for Cardiovascular Prevention \& Rehabilitation, Reiner Z, Catapano AL et al. ESC Committee for Practice Guidelines (CPG) 2008-2010 and 2010-2012 Committees. ESC/EAS Guidelines for the management of dyslipidaemias: the Task Force for the management of dyslipidaemias of the European Society of Cardiology (ESC) and the European Atherosclerosis Society (EAS). Eur Heart J. 2011;32(14):1769-818. doi: 10.1093/eurheartj/ehr158.

8. Gabrielli R, Rosati MS, Vitale $\mathrm{S}$ et al. Randomized controlled trial of remote endarterectomy versus endovascular intervention for TransAtlantic Inter-Society Consensus II D femoropopliteal lesions. J Vasc Surg 2012; 56: 1598-605.

9. Baril DT, Marone LK, Kim J et al. Outcomes of endovascular interventions for TASC II B and C femoropopliteal lesions. J Vasc Surg 2008, 48, 627-33.

10. Antoniou GA, Fisher RK, Georgiadis GS et al. Statin therapy in lower limb peripheral arterial disease: systematic review and meta-analysis. Vascul Pharmacol 2014; 63: 79-87.
11. Markel A. Statins and peripheral arterial disease. Int Angiol. 2015;34(5):416-27.

12. Poredoš P, Jezovnik MK, Kalodiki E et al. Medical management of patients with peripheral arterial disease. Int Angiol 2015;34:75-93.

13. Owens CD, Kim JM, Hevelone ND et al. An integrated biochemical prediction model of all-cause mortality in patients undergoing lower extremity bypass surgery for advanced peripheral artery disease. J Vasc Surg. 2012;56(3):686-95.

14. Galiñanes EL, Reynolds S, Dombrovskiy VY et al. The impact of preoperative statin therapy on open and endovascular abdominal aortic aneurysm repair outcomes. Vascular 2015;23:344-9. doi: 10.1177/1708538114552837.

15. Suckow BD, Kraiss LW, Schanzer A et al. Vascular Study Group of New England: Statin therapy after infrainguinal bypass surgery for critical limb ischemia is associated with improved 5-year survival. J Vasc Surg 2015;61:126-133.e1.

16. Scranton RE, Dhingra R, Lawler EV et al. A six-year study of diagnostic lower extremity imaging practice patterns and outcomes in the Veterans Affairs health care system. Int J Angiol. 2008;17(2):78-82.

17. Vogel TR, Su LT, Symons RG et al. Lower extremity angioplasty for claudication: a population-level analysis of 30-day outcomes. J Vasc Surg. 2007;45(4):762-7.

18. Westin GG, Armstrong EJ, Bang $\mathrm{H}$ et al. Association between statin medications and mortality, major adverse cardiovascular event, and amputation-free survival in patients with critical limb ischemia. J Am Coll Cardiol. 2014;63(7):682-90. doi: 10.1016/j.jacc.2013.09.073.

19. Dosluoglu HH, Davari-Farid S, Pourafkari L et al. Statin use is associated with improved overall survival without affecting patency and limb salvage rates following open or endovascular revascularization. Vasc Med 2014;19:86-93.

20. TASC Steering Committee, Jaff MR, White CJ et al. An update on methods for revascularization and expansion of the TASC lesion classification to include below-the-knee arteries: a supplement to the Inter-Society Consensus for the Management of Peripheral Arterial Disease (TASC II). J Endovasc Ther. 2015; 22(5): 663-77. doi: $10.1177 / 1526602815592206$.

21. Wieczór R, Gadomska G, Góralczyk B et al. Selected angiogenic factors in plasma of patients with lower limb symptomatic peripheral arterial disease - preliminary report. Int Angiol. 2015;34(6):545-51.

22. Laganà $\mathrm{D}$, Carrafiello $\mathrm{G}$, Barresi $\mathrm{M}$ et al. "Full metal jacket" with direct stenting of complete chronic occlusions of the superficial femoral artery. Radiol Med. 2011;116(3):444-53. doi: 10.1007/s11547-011-0614-1.

23. Shah PS, Hingorani A, Ascher E et al. Full metal jacket stenting of the superficial femoral artery: a retrospective 
review. Ann Vasc Surg. 2011;25(1):127-31. doi:

10.1016/j.avsg.2010.11.001.

Address for correspondence:

Jacek Budzyński, MD, PhD

Clinic of Vascular and Internal Diseases

Jan Biziel University Hospital No. 2

75 Ujejskiego Street

85-168 Bydgoszcz, Poland

tel./fax: +48523655148

e-mail: budz@cps.pl

Received: 20.07.2016

Accepted for publication: 12.08.2016 